\title{
Exhausted T cells in systemic lupus erythematosus patients in long-standing remission.
}

\author{
Guadalupe Lima ${ }^{1}$, Francisco Treviño-Tello ${ }^{2}$, Yemil Atisha-Fregoso ${ }^{3}$, Luis Llorente ${ }^{1}$, Hilda \\ Fragoso-Loyo $^{1}$, and Juan Jakez-Ocampo ${ }^{1}$ \\ ${ }^{1}$ Instituto Nacional de Ciencias Médicas y Nutrición Salvador Zubirán \\ ${ }^{2}$ Instituto Nacional de Ciencias Médicas y Nutrición Salvador Zubiran \\ ${ }^{3}$ Elmezzi Graduate School of Molecular Medicine
}

December 10, 2020

\begin{abstract}
Introduction The mechanisms that drives SLE remission. The aim of the present study was to measure CD4 + and CD8 + T cell exhaustion in SLE patients in prolonged remission (PR-SLE) and compared them with patients with active SLE (Act-SLE) and healthy subjects Methods We included 15 PR-SLE patients, 15 Act-SLE and 29 healthy subjects. T-cell exhaustion was determined by flow cytometry according to the expression of PD-1, Tim-3, 2B4, EOMES and T-bet in CD4 + and CD8 + T cells. Dimensionality reduction using the t-Distributed Stochastic Neighbor Embedding algorithm and Clustering Analysis was used for the identification of relevant populations. Results Percentages of CD3,$+ \mathrm{CD} 4+$ and CD8 $+\mathrm{T}$ cells were similar among groups. We identified five subpopulations of CD8 + and seven of CD4 cells. The CD $4+$ Tbet + CD $45 R O+$ cells identified in the unsupervised analysis were significantly increased in PR-SLE vs Act-SLE (median: 10.20, IQR: 1.74-30.50 vs. 1.68, IQR: 0.4-2.83; $\mathrm{p}<0.01)$. CD4+EOMES + cells were also increased in PR-SLE vs Act-SLE (5.24, IQR: 3.38-14.70 vs. 1.39, IQR: 0.48-2.87; $\mathrm{p}<0.001)$. CD8+ EOMES + cells were increased in PR-SLE vs Act-SLE (37.6, IQR: 24.9-53.2 vs 8.13, IQR: 2.33-20.5; $\mathrm{p}<0.001$ ). Exhausted and activated $\mathrm{T}$ cells presented an increased frequency of PD-1, CD57 and EOMES in SLE patients vs healthy subjects. Conclusions Some subpopulations of $\mathrm{T}$ cells expressing markers associated with exhaustion are increased in patients in remission, supporting T-cell exhaustion as a tolerance mechanism in SLE. Exhaustion of specific populations of $\mathrm{T}$ cells might represent a potential therapeutic tool that will contribute to the goal of achieving sustained remission in these patients.
\end{abstract}

\section{Exhausted T cells in systemic lupus erythematosus patients in long-standing remission .}

Short title: Exhausted T cells in SLE

Guadalupe Lima*+, Francisco Treviño-Tello*+, Yemil Atisha-Fregoso $^{++S S}$, Luis Llorente*, Hilda FragosoLoyo*[?], Juan Jakez-Ocampo*[?]

*Department of Immunology and Rheumatology, Instituto Nacional de Ciencias Medicas y Nutricion Salvador Zubiran, Mexico City, Mexico.

${ }^{++}$Elmezzi Graduate School of Molecular Medicine at Northwell Health, Manhasset, New York, USA.

${ }^{\mathrm{SS}}$ Institute of Molecular Medicine, the Feinstein Institutes for Medical Research, Manhasset, New York, USA.

${ }^{+}$These authors have contributed equally to this work.

${ }^{[?]}$ Correspondence: 
Juan Jakez-Ocampo: jjakez_ocampo@yahoo.com or Hilda Fragoso-Loyo: hfragosol@gmail.com

Key words: Clinical remission in SLE; T cell exhaustion; Clustering analysis.

Word count: 2625

\section{Abstract}

\section{Introduction}

The mechanisms that drives SLE patients to achieve remission are unknown, one possible explanation might be $\mathrm{T}$ cell exhaustion. The aim of the present study was to measure CD4+ and CD8+ T cell exhaustion in SLE patients in prolonged remission (PR-SLE) and compared them with patients with active SLE (Act-SLE) and healthy subjects

\section{Methods}

We included 15 PR-SLE patients, 15 Act-SLE and 29 healthy subjects. T-cell exhaustion was determined by flow cytometry according to the expression of PD-1, Tim-3, 2B4, EOMES and T-bet in CD4+ and CD8+ $\mathrm{T}$ cells. Dimensionality reduction using the t-Distributed Stochastic Neighbor Embedding algorithm and Clustering Analysis was used for the identification of relevant populations.

\section{Results}

Percentages of CD3+, CD4 + and CD8 + T cells were similar among groups. We identified five subpopulations of $\mathrm{CD} 8+$ and seven of $\mathrm{CD} 4+$ cells. The $\mathrm{CD} 4+$ Tbet $+\mathrm{CD} 45 \mathrm{RO}+$ cells identified in the unsupervised analysis were significantly increased in PR-SLE vs Act-SLE (median: 10.20, IQR: 1.74-30.50 vs. 1.68, IQR: 0.4-2.83; $\mathrm{p}<0.01)$. CD4+EOMES + cells were also increased in PR-SLE vs Act-SLE (5.24, IQR: 3.38-14.70 vs. 1.39, IQR: 0.48-2.87; $\mathrm{p}<0.001)$. CD8+ EOMES + cells were increased in PR-SLE vs Act-SLE (37.6, IQR: 24.953.2 vs 8.13, IQR: $2.33-20.5 ; \mathrm{p}<0.001)$. Exhausted and activated $\mathrm{T}$ cells presented an increased frequency of PD-1, CD57 and EOMES in SLE patients vs healthy subjects.

\section{Conclusions}

Some subpopulations of $\mathrm{T}$ cells expressing markers associated with exhaustion are increased in patients in remission, supporting T-cell exhaustion as a tolerance mechanism in SLE. Exhaustion of specific populations of $\mathrm{T}$ cells might represent a potential therapeutic tool that will contribute to the goal of achieving sustained remission in these patients.

\section{Introduction}

Systemic lupus erythematosus (SLE) is an autoimmune, multifactorial disease with broad tissue and organ involvement. Its manifestations are usually heterogeneous, with a fluctuating and unpredictable course [1]. Despite the advances in the treatment and understanding of its pathogenesis, disease activity continues to be one of the major causes of morbidity and mortality. Progressive accumulation of irreversible damage is attributed to chronic inflammation, as well as to the prolonged use of steroids and immunosuppressants [2]. Many studies conducted in recent years have focused on the search for treatments and interventions that reduce or prevent relapses in patients with SLE, with the goal of reaching a sustained remission and limiting damage [3,4]. Despite the information provided by these studies, very little is known about the clinical and serological features that characterize the group of patients with SLE who achieve sustained remission and the cellular, genetic, or molecular factors involved in this task.

T-cell exhaustion is a complex process that has been increasingly recognized in recent years in patients with chronic infections and cancer. T-cell exhaustion is driven by persistent TCR stimulation in the absence of enough co-stimulation and is characterized by limited proliferation, cytokine production, and effector function [5]. There is not a universally accepted set of markers that define the population of exhausted $\mathrm{T}$ cells, however, is recognized that they express a variety of inhibitory receptors that reduce their response 
after activation, such as PD-1, Tim-3 or 2B4 and differentially express transcription factors such as EOMES and T-bet.

There are few reports about the role of exhausted $\mathrm{T}$ cells in autoimmunity, but they have linked their presence with an improved prognosis in several autoimmune diseases [6]. There is also evidence of an association of exhausted T cells and increased immune tolerance in transplantation [7]. Because of this potentially favorable effect of the exhaustion process in autoimmunity, we conducted this study with the aim of measuring CD4+ and CD8+ T cell exhaustion by quantifying surface inhibitory molecules and transcriptional factors by flow cytometry in SLE patients in prolonged remission and to compare them with active SLE patients and healthy subjects.

Material and Methods

\section{Patients and controls}

This was a cross-sectional study conducted at the Instituto Nacional de Ciencias Medicas y Nutricion Salvador Zubiran, a tertiary care center in Mexico City. We included 15 patients with SLE in long-term remission without treatment (PR-SLE). Prolonged remission was defined as a clinical SLEDAI of 0 (regardless of serological activity, i.e., anti-dsDNA antibodies and/or hypocomplementemia) and being free of immunosuppressive or antimalarial treatment for at least 10 years [8]. As controls, we included 15 patients with clinically active SLE (Act-SLE) with a clinical SLEDAI 2K [?]3 (excluding hypocomplementemia and high titers of anti-dsDNA) and 29 healthy subjects (HS). All the healthy controls were confirmed negative for antinuclear antibodies and with no personal or familial history of autoimmune diseases. All patients with SLE met the SLICC 2012 classification criteria [9].

\section{Flow Cytometry}

One sample of peripheral blood was obtained from each patient according to standard procedures. EDTAtreated blood samples were analyzed by 8-color flow-cytometry (Becton Dickinson Canto II Cytometer) using several combinations of fluorescence-labeled antibodies distributed in the following panels: Panel 1: CD3 FITC, CD4 APC/Cy7, CD25 BV421, CD127 APC and FOXP3 PE; Panel 2: CD3 PerCP/Cy5, CD4 APC/Cy7, CD8 Alexa Fluor 488, HLA-DR PE, CD244 (2B4) APC, CD279 (PD-1) BV421, CD38 BV510 and PE/Cy7 CD366 (Tim-3); Panel 3: CD3 PE/Cy7, CD4 PE/Cy5, CD8 BV510, CD27 APC/Cy7, CD57 APC, CD45RO FITC, T-bet BV421 and EOMES PE; Panel 4: CD3 APC, CD4 BV510, CD8 PE, CD45RA FITC, CD197 APC/Cy7. All antibodies were purchased from Biolegend Inc. (San Diego, CA) except EOMES PE (eBioscience). Briefly, $250 \mu \mathrm{L}$ of blood was incubated with fluorochrome-conjugated antibodies for $30 \mathrm{~min}$ at room temperature prior to lysis with RBC Lysis Buffer (Biolegend Inc.) and fixation with 3\% formaldehyde in PBS.

OneFlow Setup Beads (BD Biosciences) were used to adjust instrument settings, set fluorescence compensation, and check instrument sensitivity. 'Fluorescence minus one' controls were used to determine positive and negative staining boundaries for each fluorochrome. Two hundred and fifty thousand events were recorded for each sample and analyzed with the FlowJoß software v.10.7.1 (FlowJo, LLC, Oregon, USA). FlowAI plugin was used to perform an automatic or interactive quality control on the ffes data [10]. Lymphocyte population was identified in an SSC-A versus FSC-A plot, followed by doublets exclusion in a FSC-Height (FSC-H) by FSC-Area (FSC-A) scatter plot. Phenotypes used to define mayor and minor T cell populations are depicted in Table 3.

\section{Dimensionality Reduction and Clustering Analysis}

After excluding death cells and doublets, the resulting flow cytometry data files were gated on the CD3+ population using FlowJo 10.7.1. Prior to further analysis, all groups were down-sampled to equal cell numbers and concatenated in a single file. We then used a tSNE algorithm to reduce dimensionality of the data followed by Self Organized Maps (FlowSOM) [11] for unsupervised clustering of the data into subpopulations. The resulting subpopulations were further analyzed for specific marker expression and frequency in each group. We also compared populations that have been reported in the literature and that were manually 
gated independently of the findings of the unsupervised clustering. These populations were: CD4/CD8 PD1+, CD4/CD8 T-bet+, CD4/CD8 2B4, CD4/CD8 Tim-3, CD4/CD8 EOMES, CD4/CD8 CD57, CD4/CD8 CD38+HLA-DR+ (activated T cells) and CD4/CD8 CD38+HLA-DR+PD-1+ (exhausted T cells).

\section{Statistical analysis}

Descriptive statistics: Continuous data is presented as median with interquartile ranges (IQR; 25 - 75). For comparisons of quantitative variables between 2 groups, the Mann-Whitney U test was used, while the Kruskal-Wallis test followed by Dunn's test was used to compare [?]3 groups. Categorical values are presented as number and percentage. Chi-squared test was used to compare categorical variables between groups. The statistical analysis was performed using the SPSS software v.21.0 (IBM). P value [?] 0.05 was considered significant.

The study was approved by the Institutional Review Board of the Instituto Nacional de Ciencias Medicas y Nutricion Salvador Zubiran (Ref. 2232). All subjects gave their informed consent to participate according to the Declaration of Helsinki.

\section{Results}

\section{Clinical and demographic characteristics}

Of the fifteen patients with PR-SLE, fourteen (93.3\%) were women, with a median age of 49 years (4860). All Act-SLE patients were women with a median age of 33 years (30-46) and 28 (97\%) of the healthy subjects were women, median age 43 years (32-52). Patients with Act-SLE presented lymphopenia, with lower lymphocyte counts when compared with PR-SLE and healthy controls $(1.12$ x103 / $\mu \mathrm{L}[0.74-1.71]$ vs $1.67 \times 103[1.23-2.76]$ vs $2.17 \times 103[1.61-2.41] \mathrm{p}=0.030)$ The demographic and clinical characteristics at the time of recruitment are detailed in Table 1.

No differences were found between in the SLICC 2012 classification criteria at diagnosis between both groups of patients. At inclusion, disease duration in PR-SLE patients was 25.6 years (19.1 - 35.4) vs 9.25 (5.75 $12.5)$ in Act-SLE patients $(\mathrm{p}<0.001)$. The median time in remission was 16.9 years $(12.1-20.6)$. The PRSLE group had a SLEDAI-2k score of $0(0-2)$ versus $8(6-16)$ in the Act-SLE group $(\mathrm{p}=0.001)$. The clinical manifestations, activity indices and accumulated damage, and treatment of active patients are described in Table 2 .

\section{Major Lymphocyte Populations}

There were no differences in the relative percentage of CD3+, CD4+ or CD8+ T cells between groups. Among the subpopulations of CD4+ T cells, only the central memory compartment was significantly different between groups, being expanded in the PR group, compared with the other two. More differences were observed in the subpopulations of CD8+ T cells. Similar to what we observed within CD4+ T cells, patients in PR showed an increased central memory compartment, and a concomitant reduction in the percentage of naïve CD8+ cells, when compared with the other groups. Meanwhile, patients with Act-SLE presented a decreased percentage of CD8+ effector memory cells. The percentages of the subpopulations analyzed and the $\mathrm{p}$ values for the comparisons are described in Table 3.

\section{$\mathrm{T}$ cell exhaustion markers and populations}

As there is no clear consensus, and just little information about the specific marker combination that describes specific subpopulations of exhausted T cells in patients with SLE, we initially used an unsupervised approach to explore the behavior and clustering of surface markers and transcription factors that have been reported as relevant in exhausted $\mathrm{T}$ cells (see methods for the complete list of markers included). As a first step, we generated tSNE maps using concatenated files with an equal number of CD3+ cells from all the patients included in each group. Figures 1A and 1B show the tSNE plots for each group, and the distribution of the included markers across the plots. In order to identify the clustering of these markers in subpopulations, we used a self-organizing maps algorithm [11]. We could identify five subpopulations of CD8+, seven of CD4+ cells and three CD3+CD4-CD8-. The heatmaps and tables showing the relative expression of markers in 
each cluster across all the identified subpopulations are shown in figure 1C. Overlapping of these populations on the tSNE plots are shown in 1D. Histograms represent the relative expression of a CD3+ CD8+ cell population that seems to be exclusive to the PR-SLE group, in both in panel II and III $(2.86 \%$ and $3.75 \%$ respectively) and which expresses significantly transcription factors and surface markers associated with senescence and depletion.

\section{Populations differentially represented in the PR when compared with the Act-SLE group .}

Within the CD4+ compartment, a population of Tbet+CD45RO+ cells identified in the unsupervised analysis, which represents $10.20 \%(1.74-30.50)$ of the CD4+ cells in the PR group, was significantly increased compared with the Act-SLE (1.68, IQR: 0.42-2.83) and HS (4.08, IQR: 1.64-5.48). The CD4+EOMES+ cells were also increased in the PR group compared with Act-SLE and HS. The other population that was significantly different between PR and Act-SLE was the CD4+Tbet+ cells, which were increased in the PR group, and similar to the HS.

Within the CD8+ T cells, the proportion of PD1+ cells was apparently increased only in the PR group, even though the difference was only significant when compared with the HS. The CD8+2B4+ cells, identified through the unsupervised clustering, were increased in the PR vs Act-SLE group. The most striking difference was observed in the EOMES+ cells, with a median of 37.6 (24.9-53.2) in the PR group, vs 12.9 (6.78-24.8) in the HS and 8.13 (2.33-20.5) in the Act-SLE groups.

\section{Differences between SLE patients and healthy subjects}

We observed a significant increase in the frequency of expression of cell activation and exhaustion markers in both the CD4+ and the CD8+ compartment between the SLE patients and HS group. The T cell subpopulations in SLE patients presented an increase in the frequency of PD-1, CD57, EOMES, and exhausted and activated populations when compared with the HS group. Table 4 and Figure 2 shows all the populations that were identified as differentially expressed between groups.

\section{Discussion}

Remission in SLE implies absence of major signs and symptoms, which translate into a lower probability of adverse outcomes when this state is sustained. In 2016, the conclusions from an international consensus of experts on the definitions of remission in SLE (DORIS) were published [12]. It was established that to catalog a patient in remission it is important to rely on activity indices (as SLEDAI, BILAG or ECLAM). The consensus also highlights the importance of differentiating patients in remission who are under treatment (low doses of glucocorticoids, maintenance immunosuppressants and/or biological maintenance) from those without treatment (or with antimalarials). All the patients classified and included as in remission in this study, had a sustained clinical SLEDAI of 0 , and were free of medication, including antimalarials for at least 10 years.

Patients with SLE, even after achieving remission for a long time, are not considered cured, and there is evidence confirming a subjacent autoimmune response: presence of autoantibodies or low complement [8] or relapses after long periods in remission (over 10 years) [13]. How the altered immune response is kept under control in patients with lupus that achieve remission is not understood and understudied. Our results support $\mathrm{T}$ cell exhaustion as a contributor in this process.

Using an unsupervised flow cytometric analysis, we found that multiple populations that express markers that have been associated with T cell exhaustion like PD-1, CD57 and EOMES are altered in patients with SLE when compared with HS. Remarkably, not all the populations with markers of exhaustion were different between SLE patients and HS, finding consistent with previous reports, that suggest that opposed to what occurs in models of chronic viral infection, patients with autoimmune disease have a selective upregulation of exhaustion associated receptors [6]. This phenomenon suggests that the signals that induce exhaustion in each condition are somehow context specific.

Exhausted $\mathrm{T}$ cells have a differential transcriptome and epigenome when compared with effector $\mathrm{T}$ cells [14, 
15], and exhaustion is considered a stable differentiation fate of $\mathrm{T}$ cells, even though it can be reversed by therapeutic interventions. The presence of exhausted $\mathrm{T}$ cells in patients with an active autoimmune disease, suggests that cells that are activated by an ongoing inflammatory response or by the persistent presence of an autoantigen, cannot get costimulatory signals above certain threshold, and are acquiring an exhausted phenotype [16]. The function of these cells is not completely known, but in patients with cancer, exhausted cells in the tumor microenvironment contribute with the tumor immune evasion, or induced tumor tolerance. This deleterious effect in cancer might have a positive implication in other diseases, when tolerance has a positive connotation, as transplantation and autoimmunity [17].

In patients with autoimmune diseases, $T$ cell exhaustion has been associated more with the progression than with the onset of the disease $[6,18]$. In this regard, this is the first study that identifies specific subpopulations of exhausted $\mathrm{T}$ cells in patients with lupus in long-term remission.

The mechanisms underlying the hyporesponsive state of exhausted cells are also subject of study. We found an increased frequency of CD4 and CD8 EOMES+ cells in patients in remission. This transcription factor, member of the T-bet family, has also been described as altered in chronic viral infection [19] and cancer [20]. Remarkably, in patients with cancer, EOMES is necessary for the induction of cytotoxic anti-tumor T cells, however, the deletion of one allele of this transcript factor reduced the number of exhausted $\mathrm{T}$ cells and improved tumor control [20]. This dual effect emphasizes the fine tuning involved in the generation of an exhausted versus effector response in $\mathrm{T}$ cells. A deeper understanding of the mechanisms that induce exhaustion of specific populations of $\mathrm{T}$ cells that are pathogenic in patients with SLE, might represent a potential therapeutic tool that will contribute to the goal of achieving sustained remission.

There are some limitations of this study. The study is transversal, however, to identify and study longitudinally patients in long-term remission free of medication before they achieve this status is probably not feasible. We believe this group of patients provides an ideal group for this analysis, as there is no confounding effect caused by medications and there is no recent evidence of disease activity. We studied a relatively reduced number of markers associated with exhaustion, however, these were carefully selected and allowed us to identify populations that were differentially present in the groups of interest.

Our results confirm the presence of certain subpopulations of exhausted T-cells in patients in lupus that are unequivocally in remission. This suggests a potential role of T-cell exhaustion as a mechanism of tolerance in patients with SLE. The populations identified in the present study warrant further investigation as prognostic markers in longitudinal studies of these patients, and in independent cohorts. The identification of the specific mechanisms behind the origin of these populations might provide new therapeutic options in SLE.

\section{Aknowledgements}

This research was supported by a grant from the Consejo Nacional de Ciencia y Tecnología-Fondo Sectorial de Investigación en Salud (CONACYT-FOSIS), Grant No.289732, México. This work was the Master's in Science degree thesis of Francisco Treviño-Tello, Universidad Anáhuac del Norte, México.

\section{Abbreviations}

SLE Systemic lupus erythematosus

PR-SLE SLE in long-term remission

Act-SLE Clinically active SLE

HS Healthy subjects

PD-1 Programmed cell death protein 1

Tim-3 T-cell immunoglobulin and mucin domain-3

2B4 Natural killer cell receptor

EOMES Eomesodermin 
T-bet T-box transcription factor TBX21

CD Cluster of differentiation

\section{Author contributions}

GL and FT-T performed immunological experiments, analyzed results. YA-F and LL conceived and supervised experiments, analyzed results, and wrote the manuscript. HF-L supervised and analyzed clinical data and results. JJ-O analyzed clinical data, analyzed results, and wrote the manuscript.

\section{References}

1. Tsokos, G.C., Autoimmunity and organ damage in systemic lupus erythematosus. Nat Immunol 2020; 21 : 605-4.

2. Sutton EJ, Davidson JE, Bruce IN. The systemic lupus international collaborating clinics (SLICC) damage index: a systematic literature review. Semin Arthritis Rheum 2013; 43 : 352-61.

3. Urowitz, MB, Feletar M, Bruce IN, Ibanez D, Gladman DD. Prolonged remission in systemic lupus erythematosus. J Rheumatol 2005;32 : 1467-72.

4. Zen M, Iaccarino L, Gatto M, Bettio S, Nalotto L, Ghirardello A, Punzi L, Doria A. Prolonged remission in Caucasian patients with SLE: prevalence and outcomes. Ann Rheum Dis 2015; 74 : 2117-22.

5. Wherry EJ. T cell exhaustion. Nat Immunol 2011;12 : 492-9.

6. McKinney EF, Lee JC, Jayne DRW, Lyons PA, Smith KGC. T-cell exhaustion, co-stimulation and clinical outcome in autoimmunity and infection. Nature 2015; 523 : 612-6.

7. Shahbazi M, Soltanzadeh-Yamchi M, Mohammadnia-Afrouzi M. T cell exhaustion implications during transplantation. Immunol Lett2018; 202 : 52-8.

8. Jakez-Ocampo J, Rodriguez-Armida M, Fragoso-Loyo H, Lima G, Llorente L, Atisha-Fregoso Y. Clinical characteristics of systemic lupus erythematosus patients in long-term remission without treatment. Clin Rheumatol 2020; 39 : 3365-71.

9. Petri M, Orbai A-M, Alarcon GS, Gordon C, Merrill JT, Fortin PR, Bruce IN, Isenberg D, et al. Derivation and validation of the Systemic Lupus International Collaborating Clinics classification criteria for systemic lupus erythematosus. Arthritis Rheum 2012; 64 : 2677-86.

10. Monaco G, Chen H, Poidinger M, Chen J, de Magalhaes JP, Larbi A. flowAI: automatic and interactive anomaly discerning tools for flow cytometry data. Bioinformatics 2016; 32 : 2473-80.

11. Van Gassen S, Callebaut B, Van Helden MJ, Lambrecht BM, Demeester P, Dhaene T, Saeys Y. FlowSOM: Using self-organizing maps for visualization and interpretation of cytometry data. Cytometry A 2015;87 : $636-45$.

12. van Vollenhoven R, Voskuyl A, Bertsias G, Aranow C, Aringer M, Arnaud L, Askanase A, Balažová P, et al. A framework for remission in SLE: consensus findings from a large international task force on definitions of remission in SLE (DORIS). Ann Rheum Dis 2017;76 : 554-61.

13. Tselios K, Gladman DD, Touma Z, Su J, Anderson N, Urowitz MB. Clinical Remission and Low Disease Activity Outcomes Over 10 Years in Systemic Lupus Erythematosus. Arthritis Care Res (Hoboken) 2019;71 (6):822-28.

14. Pauken KE, Sammons MA, Odorizzi PM, Manne S, Godec J, Khan O, Drake AM, Chen Z, et al. Epigenetic stability of exhausted T cells limits durability of reinvigoration by PD-1 blockade. Science $2016 ; 354: 1160-65$.

15. Sen DR, Kaminski J, Barnitz RA, Kurachi M, Gerdemann U, Yates KB, Tsao H-W, Godec J, et al., The epigenetic landscape of T cell exhaustion. Science 2016; 354 :1165-69. 
16. McKinney EF, Smith KGC. Metabolic exhaustion in infection, cancer and autoimmunity. Nat Immunol 2018;19 : 213-21.

17. Pawelec G. Is There a Positive Side to T Cell Exhaustion?Front Immunol 2019; 10 :111.

18. Long SA, Thorpe J, DeBerg HA, Gersuk V, Eddy J, Harris KM, Ehlers M, Herold KC, et al. Partial exhaustion of CD8 T cells and clinical response to teplizumab in new-onset type 1 diabetes. Sci Immunol2016; 1 : eaai7793.

19. Paley MA, Kroy DC, Odorizzi PM, Johnnidis JB, Dolfi DV, Barnett BE, Bikoff EK, Robertson EJ, et al. Progenitor and terminal subsets of CD8+ T cells cooperate to contain chronic viral infection. Science2012; $338: 1220-5$.

20. Li J, He Y, Hao J, Ni L, Dong C. High Levels of Eomes Promote Exhaustion of Anti-tumor CD8 (+) T Cells. Front Immunol 2018;9 : 2981.

Table 1. Clinical Characteristics of Prolonged Remission SLE Patients, Active SLE and Healthy Subjects.

Demographics

Sex

Female $(\%)$

Age (years)

Body Mass Index

Smoker (\%)

Laboratory characteristics

Positive ANA (\%)

ESR

Positive ESR (\%)

Hemoglobin (g/dl)

Hematocrit (\%)

Leukocytes $\left(\mathrm{x} 10^{3}\right)$

Neutrophils $\left(\mathrm{x} 10^{3}\right)$

Lymphocytes $\left(\mathrm{x} 10^{3}\right)$

Platelets $(\mathrm{K} / \mu \mathrm{L})$

n/a: not applicable. ANA: antinuclear antibodies. ESR: Erythrocyte sedimentation rate. Results for quantitative variables

Table 2. SLICC 2012 criteria, disease activity, accrual damage, and treatment in the SLE patients.

Duration of SLE (years)

Remission time (years)

SLICC 2012 SLE classification criteria items

Acute cutaneous lupus

Chronic cutaneous lupus

Nasal or oral ulcers

Alopecia

Synovitis

Serositis 
Renal

Neurologic

Autoimmune Hemolytic Anemia

Leuko-lymphopenia

Thrombocytopenia

Positive ANA

Positive anti-dsDNA

Positive anti-Smith

Antiphospholipid antibodies

Hypocomplementemia

Positive Coombs test

SLEDAI 2K

Arthritis

Hematuria

Proteinuria

Pyuria

Low complement

Increased anti-dsDNA titers

Fever

Serological SLEDAI 2K items

$\mathrm{C} 3(\mathrm{mg} / \mathrm{dL})$

Low C3 (\%)

$\mathrm{C} 4(\mathrm{mg} / \mathrm{dL})$

Low $\mathrm{C} 4(\%)$

anti-dsDNA (UI/mL)

SLICC/ACR DI

Secondary APS

Treatment

Prednisone

Dose (mg)

Immunosupressor

Azathioprine

Methotrexate

Mycophenolate mofetil

Antimalarial

n/a: not applicable. ANA: antinuclear antibodies. SLEDAI: SLE Disease Activity Index. SLICC: Systemic Lupus Internati

Table 3. CD4+ and CD8+ memory subpopulations and $\mathrm{T}$ regs.

$\mathrm{T}$ cells populations

\section{Healthy Subjects}

\section{Active SLE}

\section{PR-SLE}

p

$\mathrm{N}=\mathbf{2 9}$

$\mathrm{N}=15$ 


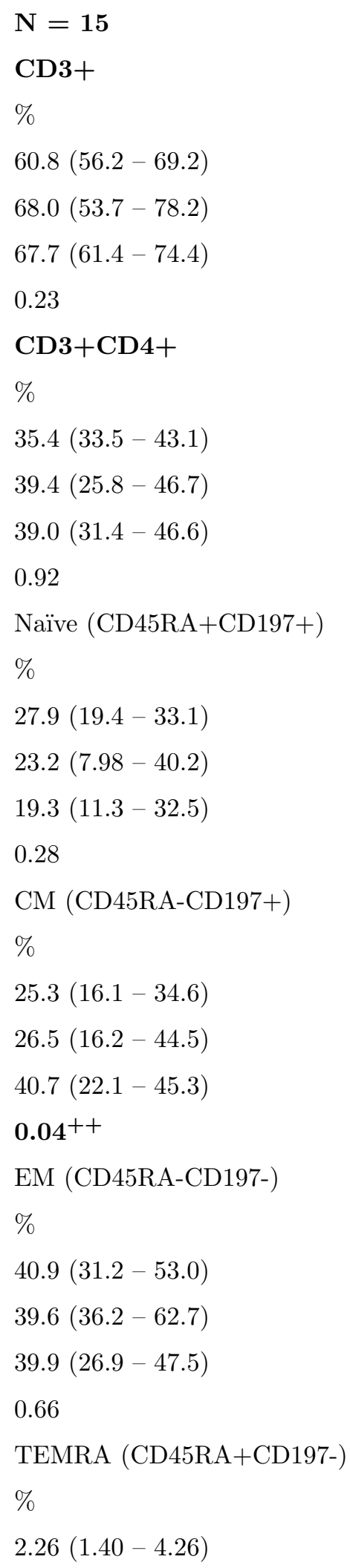




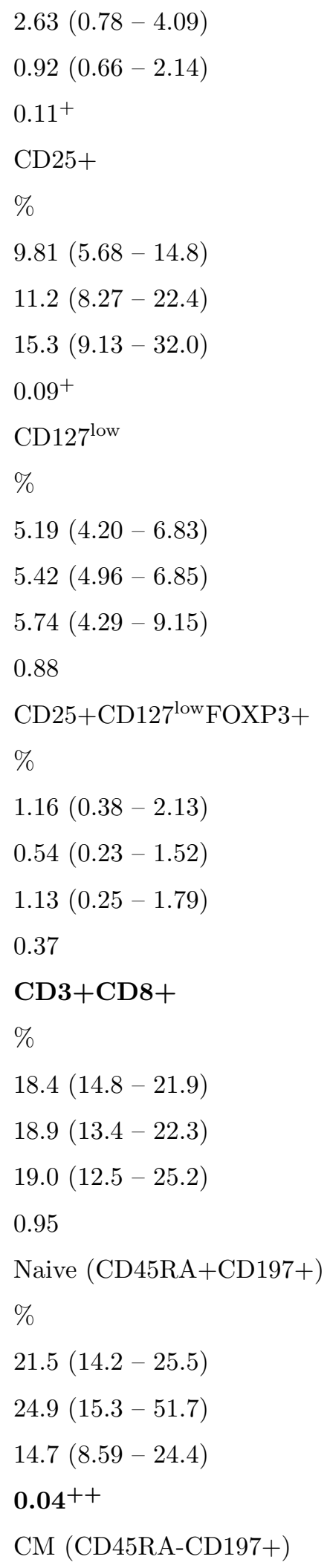


$3.13(1.71-5.67)$

$2.88(1.42-6.45)$

$8.73(3.74-10.3)$

$0.01^{++,++}$

EM(CD45RA-CD197-)

$\%$

$41.4(36.1-45.2)$

$27.8(18.0-38.8)$

$38.0(28.8-50.0)$

$0.04^{*}$

TEMRA (CD45RA+CD197-)

$\%$

$33.8(24.0-38.8)$

$34.1(19.0-45.0)$

$35.2(24.4-46.6)$

0.95

Results are shown in Median with Interquartile Range, and Kruskal Wallis test was used to perform comparisons between groups. Mann Whitney $\mathrm{U}$ test was performed to compare between two groups as a post Hoc test and are represented by the following symbols. ${ }^{*}$ Healthy Subjects vs Act SLE. ${ }^{*} \mathrm{p}<0.05 .{ }^{* *} \mathrm{p}$ $<0.01 .{ }^{* * *} \mathrm{p}<0.001$. + Healthy Subjects vs PR-SLE. $+\mathrm{p}<0.05$. $++\mathrm{p}<0.01$. $+++\mathrm{p}<0.001 .++$ Act-SLE vs PR-SLE. $++\mathrm{p}<0.05 .++++\mathrm{p}<0.01 .++++++\mathrm{p}<0.001$.

Results are shown in Median with Interquartile Range, and Kruskal Wallis test was used to perform comparisons between groups. Mann Whitney $\mathrm{U}$ test was performed to compare between two groups as a post Hoc test and are represented by the following symbols. ${ }^{*}$ Healthy Subjects vs Act SLE. ${ }^{*} \mathrm{p}<0.05 .{ }^{* *} \mathrm{p}$ $<0.01 .{ }^{* * *} \mathrm{p}<0.001 .+$ Healthy Subjects vs PR-SLE. $+\mathrm{p}<0.05 .++\mathrm{p}<0.01 .+++\mathrm{p}<0.001 .++$ Act-SLE vs PR-SLE. $++\mathrm{p}<0.05 .++++\mathrm{p}<0.01 .++++++\mathrm{p}<0.001$.

Results are shown in Median with Interquartile Range, and Kruskal Wallis test was used to perform comparisons between groups. Mann Whitney U test was performed to compare between two groups as a post Hoc test and are represented by the following symbols. ${ }^{*}$ Healthy Subjects vs Act SLE. ${ }^{*} \mathrm{p}<0.05$. ${ }^{* *} \mathrm{p}$ $<0.01 .{ }^{* * *} \mathrm{p}<0.001 .+$ Healthy Subjects vs PR-SLE. $+\mathrm{p}<0.05 .++\mathrm{p}<0.01 .+++\mathrm{p}<0.001 .++$ Act-SLE vs PR-SLE. $++\mathrm{p}<0.05 .++++\mathrm{p}<0.01 .++++++\mathrm{p}<0.001$.

Results are shown in Median with Interquartile Range, and Kruskal Wallis test was used to perform comparisons between groups. Mann Whitney $\mathrm{U}$ test was performed to compare between two groups as a post Hoc test and are represented by the following symbols. ${ }^{*}$ Healthy Subjects vs Act SLE. ${ }^{*} \mathrm{p}<0.05 .{ }^{* *} \mathrm{p}$ $<0.01 .{ }^{* * *} \mathrm{p}<0.001 .+$ Healthy Subjects vs PR-SLE. $+\mathrm{p}<0.05 .++\mathrm{p}<0.01 .+++\mathrm{p}<0.001 .++$ Act-SLE vs PR-SLE. $++\mathrm{p}<0.05 .++++\mathrm{p}<0.01 .++++++\mathrm{p}<0.001$.

Results are shown in Median with Interquartile Range, and Kruskal Wallis test was used to perform comparisons between groups. Mann Whitney $\mathrm{U}$ test was performed to compare between two groups as a post Hoc test and are represented by the following symbols. ${ }^{*}$ Healthy Subjects vs Act SLE. ${ }^{*} \mathrm{p}<0.05 .{ }^{* *} \mathrm{p}$ 
$<0.01 .{ }^{* * *} \mathrm{p}<0.001 .+$ Healthy Subjects vs PR-SLE. $+\mathrm{p}<0.05 .++\mathrm{p}<0.01 .+++\mathrm{p}<0.001 .++$ Act-SLE vs PR-SLE. $++\mathrm{p}<0.05$. $++++\mathrm{p}<0.01 .++++++\mathrm{p}<0.001$.

Results are shown in Median with Interquartile Range, and Kruskal Wallis test was used to perform comparisons between groups. Mann Whitney U test was performed to compare between two groups as a post Hoc test and are represented by the following symbols. * Healthy Subjects vs Act SLE. ${ }^{*} \mathrm{p}<0.05$. ** p $<0.01 .{ }^{* * *} \mathrm{p}<0.001 .+$ Healthy Subjects vs PR-SLE. $+\mathrm{p}<0.05 .++\mathrm{p}<0.01 .+++\mathrm{p}<0.001 .++$ Act-SLE vs PR-SLE. $++\mathrm{p}<0.05$. $++++\mathrm{p}<0.01 .++++++\mathrm{p}<0.001$.

Table 4. T cell exhaustion in CD4+ and CD8+ lymphocytes.

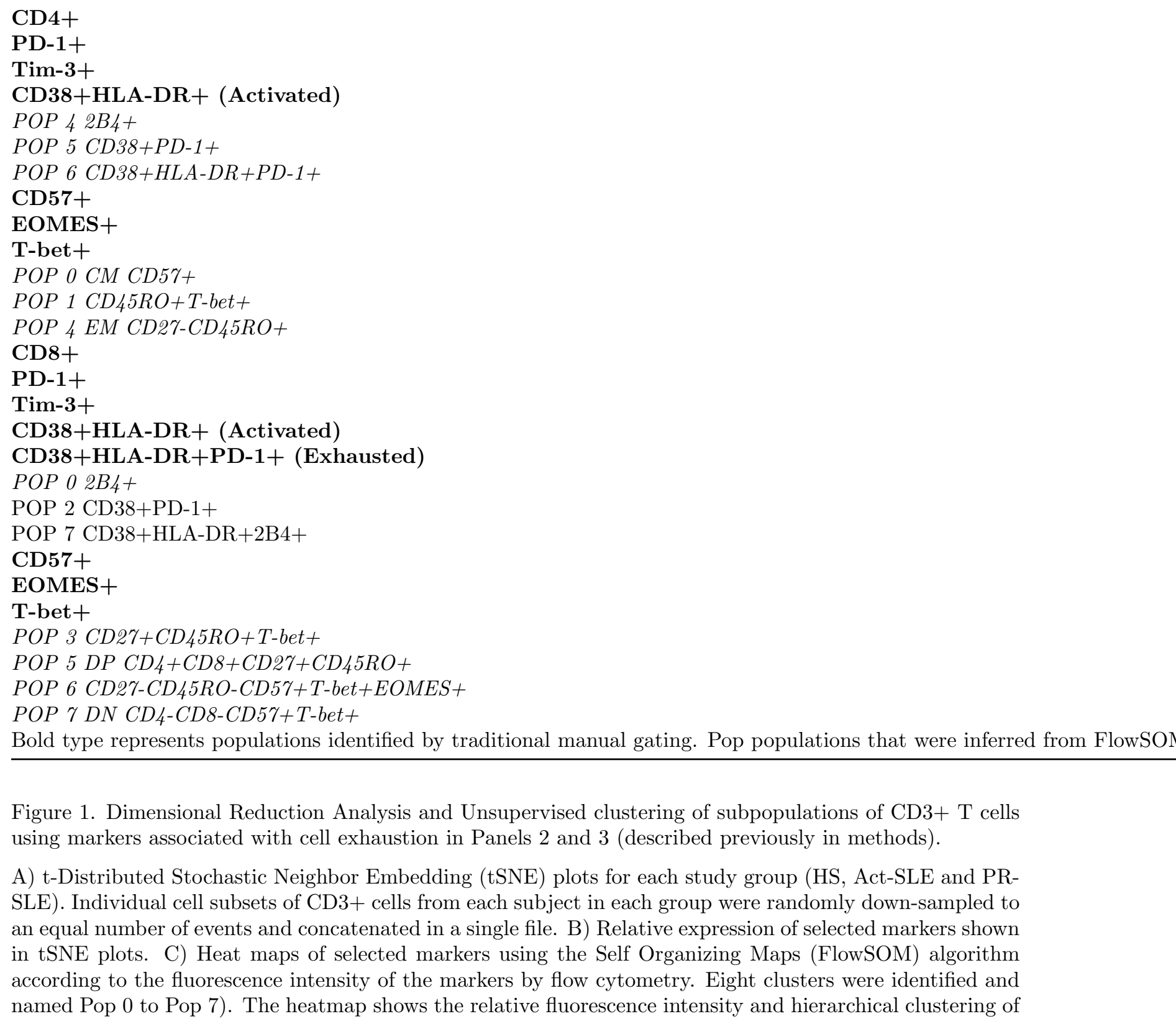

Figure 1. Dimensional Reduction Analysis and Unsupervised clustering of subpopulations of CD3+ T cells using markers associated with cell exhaustion in Panels 2 and 3 (described previously in methods).

A) t-Distributed Stochastic Neighbor Embedding (tSNE) plots for each study group (HS, Act-SLE and PRSLE). Individual cell subsets of CD3+ cells from each subject in each group were randomly down-sampled to an equal number of events and concatenated in a single file. B) Relative expression of selected markers shown in tSNE plots. C) Heat maps of selected markers using the Self Organizing Maps (FlowSOM) algorithm according to the fluorescence intensity of the markers by flow cytometry. Eight clusters were identified and named Pop 0 to Pop 7). The heatmap shows the relative fluorescence intensity and hierarchical clustering of 
each of the markers included in the subpopulations generated. Percentage relative to CD3+ cells is shown in tables below. D) Distribution of clusters identified in C overlaid on the t-SNE plots in each study group. In both panels, a CD8+ subpopulation that expresses exhaustion and senescence markers was identified exclusively in the PR-SLE group. Overlaid histograms (HS: green; Act-SLE: red; PR-SLE: purple) show relative frequencies of most abundant markers.

Figure 2. Bar graphs showing the frequencies (mean \pm SEM) of lymphocytes populations derived from traditional manual gating and FlowSOM clusters identified in the heatmap in panel a. Mann Whitney U test was performed to compare between two groups and differences are represented by the following symbols: ${ }^{*} \mathrm{p}$ $<0.05 .{ }^{* *} \mathrm{p}<0.01 .{ }^{* * *} \mathrm{p}<0.001 .{ }^{* * * *} \mathrm{p}<0.0001$. HS (healthy subjects); Act-SLE (active SLE); PR-SLE (prolonged remission SLE).
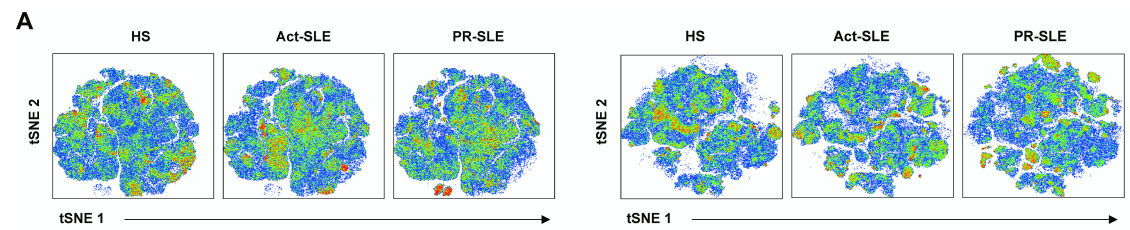

B
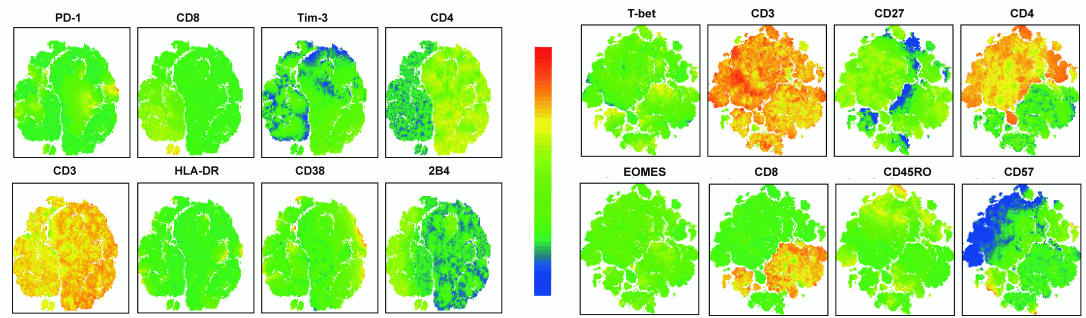

C
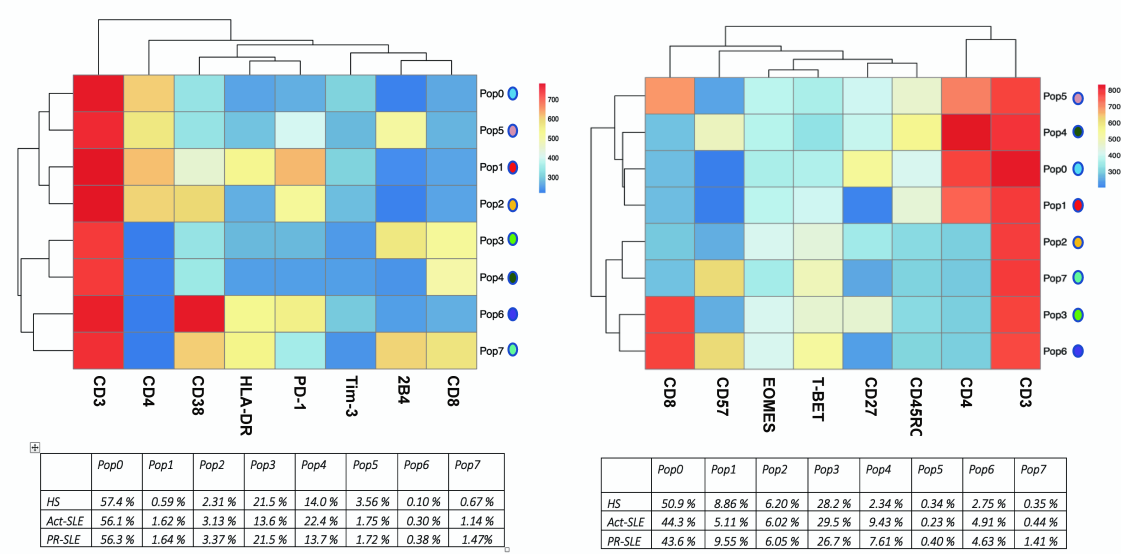

D
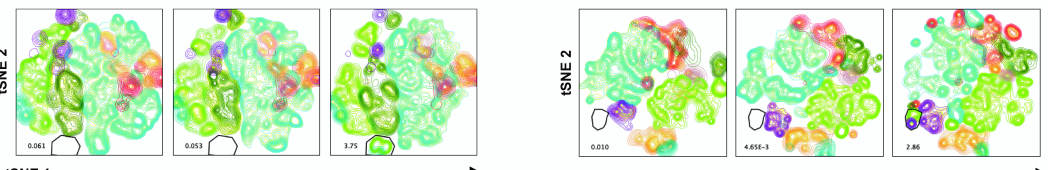

TSNE 1
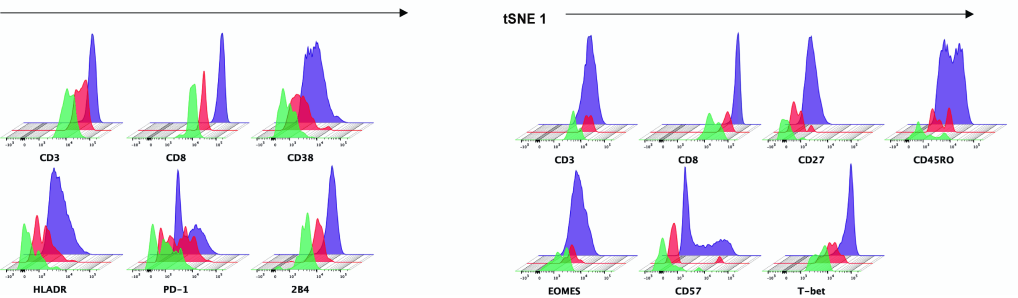

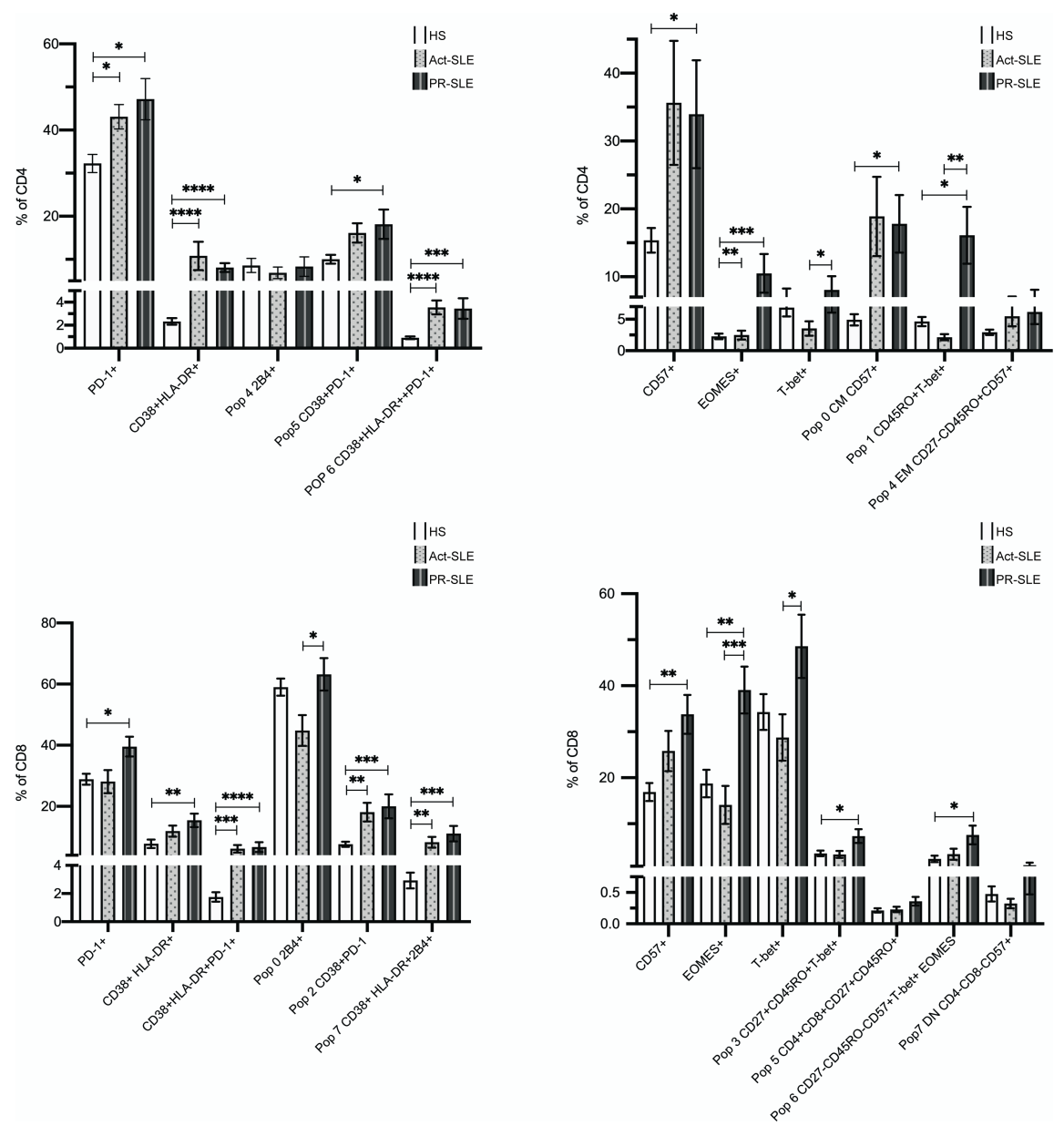